\section{Advances in brain metastases presented at the American Society of Clinical Oncology 2016 Annual Meeting: Part I}

Lucy F Chen ${ }^{*}$, , Jyoti D Patel ${ }^{2}$ \& Rimas V Lukas ${ }^{3}$

American Society of Clinical Oncology (ASCO) Annual Meeting, Chicago, IL, USA, 3-7 June 2016

The American Society of Clinical Oncology Annual Meeting took place in Chicago, IL, USA from 3 to 7 June 2016. Over 30,000 oncologists, researchers, related professionals and advocates participated in the conference which covered all aspects of oncology. An overview of the key studies in brain metastases presented at the 2016 American Society of Clinical Oncology Annual Meeting is highlighted here. This report highlights biology, epidemiology, prognosis and treatment sequelae of brain metastases.

First draft submitted: 18 July 2016; Accepted for publication: 17 August 2016; Published online: 5 September 2016

\section{Biology, epidemiology \& prognosis}

A number of studies helped clarify the incidence and prevalence of brain metastases as well as their realworld management. Some of these are single institution studies conducted at academic medical centers and may not generalize to the community oncology setting. Others have utilized national databases to help provide a more detailed picture of brain metastases incidence categorized by the underlying tumor histology. A large retrospective study from the University of Vienna reviewed charts for 2419 solid tumor brain metastases patients from 1990 to 2011. Lung cancer (43.2\%) was the most common primary tumor. This was followed by melanoma $(16.4 \%)$, breast cancer $(15.7 \%)$, colorectal $(9.3 \%)$ and renal cell carcinoma (9.1\%). A number of less commonly encountered histologies were also reported. Almost half of all patients in the cohort had only a single brain metastasis with about a quarter having more than three. This finding is somewhat surprising and may merely be a result of the bias inherent in retrospective studies. However, if earlier detection truly identifies a greater number of patients with few brain metastases, this could translate into more opportunities for focal therapy options. Multiple brain metastases were most often seen in breast cancer and least often in colorectal cancer. The shortest lagtime between initial diagnosis and diagnosis of brain metastases was seen in lung cancer and the longest was in breast cancer [1]. These points will be important to consider in both, the day-to-day clinical management of patients, as well as the design and interpretation of therapeutic trials in these patients.

The National Cancer Database was queried for the incidence of brain metastases from non-smallcell lung cancer (NSCLC) at the time of diagnosis. Of the 504,151 patients with all stages of NSCLC

\section{KEYWORDS}

- brain metastases • cancer metastases • chemotherapy

- immunotherapy

- radiotherapy • targeted therapies 
in the database between 2010 and 2013, 9.4\% were recorded as having brain metastases. Median overall survival (OS) for these patients was 6.08 months [2]. This is very similar to the median OS (7.0 months) reported in the disease specificgraded prognostic assessment prognostication system [3]. Multivariate analysis demonstrated that increasing age, adenocarcinoma histology, tumor $>3 \mathrm{~cm}$, tumor grade $\geq \mathrm{II}$ and node-positive disease increased the risk of synchronous diagnosis of brain metastases [2].

A study of the $36 \%$ of NSCLC patients with brain metastases in the IMPACT/COMPACT clinical trial on univariate analysis found that the patients with brain metastases were younger, Caucasian, were initially diagnosed with stage IV disease, had adenocarcinoma histology and had EGFR mutations. Multivariate analyses were not reported [4]. Another study of tumor tissue tested at two different institutions demonstrated a similar association on multivariate analysis between incidence of brain metastases at initial diagnosis and EGFR mutations (odds ratio: $11.8 ; \mathrm{p}=0.0002$ ). Interestingly, there was no significant difference in OS between patients with $E G F R$ mutations with or without brain metastases, although a trend toward longer survival was seen in those without brain metastases [5]. A single institution study of patients with brain metastases comparing EGFRmutated or $A L K$-translocated NSCLC patients to $E G F R / A L K$ wild-type NSCLC patients demonstrated a better OS (19.3 vs 9.7 months) in the mutated/translocated group. In this study, while the number of brain metastases influenced outcome in EGFR/ALK wild-type patients, it had no impact on the mutated/translocated patients [6]. However, a different single institution study reported that NSCLC patients with $E G F R$-mutated or $A L K$-translocated NSCLC patients with brain metastases still have a shorter OS (2.1 years) compared with the EGFR/ALKdriven patients without brain metastases (4.0 years) [7]. These outcomes appear superior to those reported for NSCLC patients in contemporary prognostication systems. In turn, the mutational/translocational status will be important to consider as we refine our existing prognostication systems. A growing understanding of the mutational status and its effects on the incidence, natural history and treatment responses of brain metastases will have the potential to impact future investigations and the clinical care of patients. This understanding in conjunction with our knowledge of CNS activity of existing and future therapeutic agents will be central to the management of patients with brain metastases [8].

A large German study looked at the management of patients with breast cancer brain metastases since 2000. Median age at diagnosis of brain metastases was 51 years old. HER 2 positivity was seen in $49.0 \%$ of patients, triple-negative $20.5 \%$ and the remainder were luminal primary $(30.5 \%)$. Median OS for the entire group was 7.1 months with a longer survival (12.1 months) in the HER2 ${ }^{+}$and shorter (4.1 months) in the triple-negative. The use of HER2-directed therapies after the diagnosis of brain metastases correlated with improved survival. This study, however, was not designed to detect a causal relationship [9]. It is important to note that while new drug treatments have been added to our breast cancer armamentarium, patients treated between 2000 and 2006 and those treated in 2007-2012 had no survival differences. This was true regardless of the breast cancer subtype highlighting the need for further development of treatment strategies for brain metastases.

The improved survival with HER2-targeted therapy after brain metastases diagnosis was supported by another study focusing on $H E R 2^{+}$ patients. This study also demonstrated longer time from initial diagnosis to development of brain metastases in the more contemporary cohorts [10]. A single institution retrospective study of breast cancer patients with brain metastases from 2007 to 2014 reported that the previously discussed disease specific-graded prognostic assessment prognostication score, as well as the number of brain metastases (1 vs $2-3$ vs $>3$ ) and the treatment patients received correlated with survival outcomes. Patients who received local therapy (surgery, stereotactic radiosurgery [SRS]) and/or chemotherapy lived longer [11]. The association between treatment and survival may solely reflect the selection bias inherent in choosing patients with more favorable prognoses for more aggressive therapeutic approaches.

While metastatic disease to the brain from gastrointestinal primaries is uncommon, a French study evaluating histologically diagnosed brain metastases from gastrointestinal primary tumors noted that the majority of tumors (95.8\%) represented adenocarcinomas. The majority of the adenocarcinomas were of colorectal origin $(71.9 \%)$ and a smaller percentage were from gastroesophageal $(17.1 \%)$ or 
biliary (7.6\%) etiologies. While the median time between initial diagnosis and brain metastases diagnosis was 25.9 months, a wide range was observed (1.3-243.2 months). Median OS was 6.6 months from the time of brain metastases diagnosis. Over half of patients received wholebrain radiotherapy (WBRT; 54.1\%), with a smaller percentage undergoing surgical resection $(17.6 \%)$ or SRS (16.0\%). While median OS was better in patients who underwent surgical resection, this may merely reflect selection bias [12]. As our understanding of the epidemiology of brain metastases progresses we will be able to provide better patient counseling, make more nuanced decisions in therapeutic management and design clinical trials with a higher likelihood of successfully answering key questions regarding patients with brain metastases.

\section{Treatment sequelae}

Venous thromboembolism (VTE) is common in patients with brain metastases occurring in about one in five patients [13]. Low-molecularweight heparin was established to be superior over warfarin in the treatment of VTE in cancer patients in the pivotal CLOT trial [14]. Intracranial hemorrhage ( $\mathrm{ICH}$ ) occurs frequently in patients with brain metastases but the true incidence is lacking as this is not routinely followed in national/regional databases. Data show that therapeutic anticoagulation is not correlated to an increased risk for ICH [13]. A single institution database identified consecutive patients with brain metastases from 2006 to 2014. One hundred and twenty-five brain metastases patients were included in the analysis which included $51.2 \%$ primary NSCLC, $12 \%$ breast and $7.2 \%$ melanoma and small-cell lung cancer. There were 9.6\% of patients with ICH but neither the site of the primary tumor nor the number of brain metastases was correlated with the development of ICH. The data from this study confirm previous findings that anticoagulation does not increase risk for ICH. It is important to note that patients treated with SRS + WBRT were more likely to develop ICH ( $\mathrm{p}=0.0014)$ [15]. Additionally, this finding was independent of anticoagulation therapy. These data further support the ASCO guidelines that brain metastases are not a contraindication for the treatment of VTE. However the finding of increased ICH with SRS + WBRT, regardless of anticoagulation therapy, will be an important factor to consider in the treatment of brain metastases patients.

\section{Summary}

Several studies highlighted here provide important considerations in the clinical management of patients with brain metastases. As we continue to better our understanding of tumor subtypes and mutational status, we will continue to improve our ability to not only treat but also counsel patients in this disease.

\section{Financial \& competing interests disclosure}

The authors have no relevant affiliations or financial involvement with any organization or entity with a financial interest in or financial conflict with the subject matter or materials discussed in the manuscript. This includes employment, consultancies, honoraria, stock ownership or options, expert testimony, grants or patents received or pending, or royalties.

No writing assistance was utilized in the production of this manuscript.

\section{References}

1 Berghoff AS, Schur S, Fuereder L et al. Descriptive analysis of 2419 patients with brain metastases of solid cancers: a real life cohort. J. Clin. Oncol. 34(Suppl.), Abstract 2072 (2016).

2 Wagar SN, Sampson PP, Robinson CG et al. Prevalence, clinical risk factors and outcomes in patients with lung cancer presenting with brain metastases. J. Clin. Oncol. 34(Suppl.), Abstract 2075 (2016).

3 Sperduto PW, Kased N, Roberge D et al. Summary report on the graded prognostic assessment: an accurate and facile diagnosisspecific tool to estimate survival for patients with brain metastases. J. Clin. Oncol. 30 (4), 419-425 (2012).
4 Turner S, Alizadeh M, Leigh N et al. Effect of molecular profile in non-small cell lung carcinoma on the development of brain metastases. J. Clin. Oncol. 34(Suppl.), Abstract e20640 (2016).

5 Prabhash K, Bhatt VR, D'Souza SP et al. Effect of EGFR mutations on outcomes in NSCLC with brain metastases. J. Clin. Oncol. 34(Suppl.), Abstract e20644 (2016).

6 Balasubramanian SK, Venur VA, Chao ST et al. Impact of $E G F R$ and $A L K$ mutation on the outcomes of non-small cell lung cancer (NSCLC) patients with brain metastases. J. Clin. Oncol. 34(Suppl.), Abstract 2005 (2016).

7 Doherty M, Korpanty G, Tomasini P et al. Effect of brain metastases on survival and systemic treatment of EGFR/ALK-driven non-small cell lung cancer. J. Clin. Oncol. 34(Suppl.), Abstract e20527 (2016).

8 Lukas RV, Kumthekar P, Rizvi S, Salgia R. Systemic therapies in the treatment of non-small cell lung cancer brain metastases. Future Oncol. 12(8), 1045-1058 (2016).

9 Mueller V, Loibl S, Laakmann E et al. Brain Metastases in Breast Cancer Network Germany (BMBC, GBG 79): treatment patterns and clinical outcome of more than 1000 patients with brain metastases from breast cancer. J. Clin. Oncol. 34(Suppl.), Abstract 2070 (2016).

10 Mounsey L, Deal AM, Benbow JM et al. A changing natural history of HER2-positive breast cancer metastatic to the brain in the era 
CONFERENCE REPORT Chen, Patel \& Lukas

of new, targeted therapies. J. Clin. Oncol. 34(Suppl.), Abstract 584 (2016).

11 Aceituno JL, Stradella A, Simon SP et al. Breast-GPA and type of treatment predictors of survival in brain metastasis patients. J. Clin. Oncol. 34(Suppl.), Abstract e13530 (2016).

12 Portales F, Bouche O, Thezenas $S$ et al. The ARCAD METACER national cohort study of brain metastases in gastro-intestinal cancers.
J. Clin. Oncol. 34(Suppl.), Abstract 2073 (2016).

13 Donato J, Campigotto F, Uhlmann E et al. Intracranial hemorrhage in patients with brain metastases treated with therapeutic enoxaparin: a matched cohort study. Blood 126, 494-499 (2015).

14 Lee AY, Levine MN, Baker RI et al. Low-molecular-weight heparin versus a coumadin for the prevention of recurrent venous thromboembolism in patients with cancer. N. Engl. J. Med. 349(2), 146-153 (2003).

15 Gruhl JD, Horstman H, Smith L et al. Complications of anticoagulant therapy in patients with brain metastases. J. Clin. Oncol. 34(Suppl.), Abstract 2078 (2016). 